\title{
Lessons from a blind study of simulated lenses: image reconstructions do not always reproduce true convergence
}

\author{
Philipp Denzel, ${ }^{1,2}$ * Sampath Mukherjee, ${ }^{3}$ Jonathan P. Coles, ${ }^{4}$ Prasenjit Saha ${ }^{1,2}$
}

\author{
${ }^{1}$ Institute for Computational Science, University of Zurich, 8057 Zurich, Switzerland \\ ${ }^{2}$ Physics Institute, University of Zurich, 8057 Zurich, Switzerland \\ ${ }^{3}$ STAR Institute, Quartier Agora - Allée du six Août, 19c B-4000 Liège, Belgium \\ ${ }^{4}$ Physik-Department, Technische Universität München, James-Franck-Str. 1, 85748 Garching, Germany
}

\begin{abstract}
In the coming years, strong gravitational lens discoveries are expected to increase in frequency by two orders of magnitude. Lens-modelling techniques are being developed to prepare for the coming massive influx of new lens data, and blind tests of lens reconstruction with simulated data are needed for validation. In this paper we present a systematic blind study of a sample of 15 simulated strong gravitational lenses from the EAGLE suite of hydrodynamic simulations. We model these lenses with a free-form technique and evaluate reconstructed mass distributions using criteria based on shape, orientation, and lensed image reconstruction. Especially useful is a lensing analogue of the Roche potential in binary star systems, which we call the lensing Roche potential. This we introduce in order to factor out the well-known problem of steepness or masssheet degeneracy. Einstein radii are on average well recovered with a relative error of $\sim 5 \%$ for quads and $\sim 25 \%$ for doubles; the position angle of ellipticity is on average also reproduced well up to $\pm 10^{\circ}$, but the reconstructed mass maps tend to be too round and too shallow. It is also easy to reproduce the lensed images, but optimizing on this criterion does not guarantee better reconstruction of the mass distribution.
\end{abstract}

Key words: Gravitational lensing: strong - methods: numerical — Galaxy: fundamental parameters - Galaxy: structure.

\section{INTRODUCTION}

Since the first discovery of a strongly lensing galaxy (Walsh et al. 1979) these systems have been used to study a range of scientific questions: the dark-matter (DM) density profile in galaxies and possible substructure (see Koopmans \& Treu 2003; Treu \& Koopmans 2004; Read et al. 2007; Koopmans et al. 2009; Auger et al. 2010; Barnabè et al. 2011; Nierenberg et al. 2017), the star formation efficiency and stellar mass function (see Leier et al. 2011, 2016; Küng et al. 2018), cosmological parameters (Paraficz \& Hjorth 2010; Suyu \& Halkola 2010; Sereno \& Paraficz 2014; Suyu et al. 2017), and the structure of lensed active galactic nuclei (see Sluse et al. 2012; Tomozeiu et al. 2018; Hutsemékers et al. 2019).

In the coming years many more lens discoveries are anticipated, increasing the number of galaxy lenses from $\sim 10^{3}$ to $\sim 10^{5}$ (Oguri \& Marshall 2010). To prepare for this

^ Email: phdenzel@physik.uzh.ch stream of new gravitational lensing data, it is important to have the tools to process information efficiently and correctly. Recent work to this end mainly aims to improve the efficiency with which gravitational lenses are analysed. In the verification of lens candidates, machine-learning-based projects and citizen science programs have been established, working concurrently, as well as complementary, to confirm and select interesting objects (Lanusse et al. 2017; Jacobs et al. 2017; Marshall et al. 2016; More et al. 2016). Since the numbers of gravitational lenses will be too high for experts to model all by themselves, efforts have been made to create crowd-sourced lens-modelling tools such as SpaghettiLens by Küng et al. (2015); Küng (2018), or automatic modellers like AutoLens by Nightingale et al. (2018), and the machine-learning code Ensai by Hezaveh et al. (2017).

Since all the aforementioned scientific questions require reconstruction of the mass distributions of lenses, it is important for lens-modelling techniques to be rigorously tested. It is an intrinsic problem of gravitational lensing that many 



Figure 1. An example of the degeneracy of lens reconstructions. The two lower panels show two different surface mass distributions (black lines indicate convergence $\kappa=1$ ). The upper panels show lensed images produced by these mass maps. The projected images are still very similar to each other, even though the mass maps show clear differences in both shape and slope. This illustrates that many different mass distributions may result in the same projected image configuration.

plausible mass distributions are able to explain observed data. This was actually pointed out in the very first paper on lens modelling (Young et al. 1981) and is generally known as lensing degeneracy (see Saha 2000). Some degeneracies can be removed by adding additional information such as kinematics, but there exist complex degeneracies (Saha \& Williams 2006; Schneider \& Sluse 2014) whose systematic effect on modelling has yet to be explored. Figure 1 illustrates the sometimes surprising character of lensing degeneracies. It is clear that blind tests of lens modelling with simulated data are needed. Such tests have already been performed on cluster lenses in the format of a challenge in which 10 groups participated and tested their various modelling methods (Meneghetti et al. 2017).

The technique of free-form lens reconstruction is a response to the problem of lensing degeneracies. Free-form lens modelling deliberately uses underconstrained models and explores lensing degeneracies in a high-dimensional parameter space. The lens mass distribution is built out of a superposition of a large number of small components, with only few assumptions about the global properties of the distribution, such as smoothness, non-negative masses, no extra images, or being centrally concentrated. Early free-form models used regularization (Saha \& Williams 1997), but a better strategy is to generate a whole ensemble of models which all fit the data with a range of different mass distributions (Williams \& Saha 2000; Saha \& Williams 2004; Lubini \& Coles 2012). If the parameter space is properly defined and sampled, the possibility is high that the true solution is contained within the ensemble model. A drawback of such solution sampling strategies is that ensembles often also contain physically unrealistic models which are in conflict with our current understanding of galaxy evolution. Such unrealistic models might bias the ensemble average, and post-processing with further non-linear constraints is then required to filter the ensemble.

This paper explores methods to evaluate and test the quality of ensemble models from (free-form) lens-modelling tools, and specifically GLASS (Coles et al. 2014) in a blind study. In a first phase, we reconstructed 15 lenses from the EAGLE suite (Evolution and Assembly of GaLaxies and their Environment; Schaye et al. 2014) simulated with SEAGLE (Simulating EAGLE LEnses) by Mukherjee et al. (2018), unaware of the actual mass distribution of the lenses. We investigated the mapping properties between source and lens plane of the individual models in the ensemble by creating synthetic images, which are recovered by using the lensing data to reconstruct the source plane and reprojecting back on to the lens plane. The quality of the data was then evaluated by comparing the synthetic images to the original data. Moreover, the arrival-time surfaces and mass distribution maps of each model in the model ensembles were visually inspected and evidently flawed models were filtered out. In a second phase, three mass distributions were unveiled and compared with the models. The three lenses were chosen to cover most distinguishing key properties of all lenses such as doubles, quads, and visible maxima. The reason for only unveiling three lenses in the beginning was to be able to improve on the rest of the lens set. In the third phase, all lens reconstructions were revised and finally compared with the simulated mass distributions.

This paper is structured as follows: Section 2 gives an overview of the theoretical framework necessary to understand the methods detailed in Section 3. In Section 4. we report on the results obtained during the analysis in the different phases, and in Section 5 the results are discussed and summarized.

\section{THEORY}

\subsection{Arrival-time surface}

Lensing can be expressed as arrival times of paths from the source to the observer which are extremal when the path corresponds to an actual light ray according to Fermat's principle. For a source at $\boldsymbol{\beta}$ the arrival time for light coming to the observer from position vector $\boldsymbol{\theta}$ on the sky, is (following Blandford \& Narayan 1986)

$t(\boldsymbol{\theta})=\frac{1+z_{L}}{2} \frac{D_{L} D_{S}}{c D_{L S}}(\boldsymbol{\theta}-\boldsymbol{\beta})^{2}-\left(1+z_{L}\right) \frac{8 \pi G}{c^{3}} \nabla^{-2} \Sigma(\boldsymbol{\theta})$

where $D_{L}, D_{S}$, and $D_{L S}$ are angular diameter distances from observer to lens, observer to source, and lens to source, $z_{L}$ is the redshift of the lens, $\Sigma(\boldsymbol{\theta})$ the sky-projected density of the lens, and $\nabla^{-2}$ is an inverse Laplacian in $2 \mathrm{D}$. The expression (1) depends, through the distances, on both source and lens redshifts. In cluster lensing, there are typically multiple sources at different redshift and sometimes multiple lens redshifts as well. In galaxy lensing, it is very rare to have more than a single lens and a single source. 
Hence, it is convenient to take out the redshift dependence and consider a scaled arrival time without the dependence on the redshifts. Writing

$$
\begin{aligned}
\Sigma(\boldsymbol{\theta}) & =\frac{c^{2}}{4 \pi G} \frac{D_{L} D_{S}}{D_{L S}} \kappa(\boldsymbol{\theta}) \\
t(\boldsymbol{\theta}) & =\left(1+z_{L}\right) \frac{D_{L} D_{S}}{c D_{L S}} \tau(\boldsymbol{\theta})
\end{aligned}
$$

and discarding the constant $\frac{1}{2} \beta^{2}$ we have

$\tau(\boldsymbol{\theta})=\frac{1}{2} \theta^{2}-2 \nabla^{-2} \kappa(\boldsymbol{\theta})-\boldsymbol{\theta} \cdot \boldsymbol{\beta}$.

The dimensionless surface density $\kappa(\boldsymbol{\theta})$ is known as the convergence. Through equation (2) $\kappa=1$ corresponds to a critical surface density (for circular lenses, this lies within the Einstein radius) which characterizes the lens system. The function $\tau(\boldsymbol{\theta})$ is known as the arrival-time surface. It is not itself observable, but still very useful since other relations can be derived from it (see e.g. Courbin et al. 2002). In particular, the lens equation

$$
\nabla \tau(\boldsymbol{\theta})=0
$$

is just Fermat's principle applied to the arrival time while the inverse magnification tensor equals the matrix of second derivatives of $\tau(\boldsymbol{\theta})$.

\subsection{Lensing Roche potential}

We now introduce a further insightful quantity. Let us rewrite the arrival time (3) slightly as

$$
\begin{aligned}
\tau(\boldsymbol{\theta}) & =\mathcal{P}(\boldsymbol{\theta})-\boldsymbol{\theta} \cdot \boldsymbol{\beta} \\
\mathcal{P}(\boldsymbol{\theta}) & =\frac{1}{2} \theta^{2}-2 \nabla^{-2} \kappa(\boldsymbol{\theta}) \\
& =2 \nabla^{-2}(1-\kappa(\boldsymbol{\theta})) .
\end{aligned}
$$

The term $\mathcal{P}$ amounts to solving a 2D Poisson for a potential and then adding a centrifugal term. That is reminiscent of the Roche potential in celestial mechanics, and so we will call $\mathcal{P}$ the lensing Roche potential. It is like the arrival-time surface without the tilting term $\boldsymbol{\theta} \cdot \boldsymbol{\beta}$, and it represents the arrival-time surface for a source at the coordinates origin. The lensing Roche potential also amounts to solving Poisson's equation with $1-\kappa$ as the notional source.

Consider what happens if we multiply $1-\kappa$ by a constant factor $\lambda$. This changes the steepness of the mass distribution. Then, $\mathcal{P}$ will also get multiplied by $\lambda$. If $\boldsymbol{\beta}$ is simultaneously multiplied by $\lambda$, the net result is to multiply $\tau$ by $\lambda$. This operation leaves the extremal points unchanged. That means the images are also unchanged, except that the overall magnification gets multiplied by $1 \lambda$ because we have rescaled the whole source plane. This is the steepness degeneracy (also called the mass-sheet degeneracy). In fact, changing $\tau(\boldsymbol{\theta})$ in a way that does not affect the extremal points will not change the images, so there are really infinitely many degeneracies (Saha 2000). But the steepness degeneracy is the simplest and most severe. The advantage of $\mathcal{P}$ is that the steepness degeneracy only multiplies it by a constant, and does not change its shape. Hence, a normalized lensing Roche potential offers a convenient way of comparing different models with the effect of the steepness degeneracy taken out.

\subsection{Lensing mass quadrupole}

Apart from the Einstein radius, which measures the total mass in the lensing region, the shape and orientation of the lensing mass are also of interest. We can probe these by considering the $2 \mathrm{D}$ inertia tensor

$I=\frac{1}{\pi}\left(\begin{array}{cc}\int \kappa(\boldsymbol{\theta}) \theta_{x}^{2} \mathrm{~d} \boldsymbol{\theta} & \int \kappa(\boldsymbol{\theta}) \theta_{x} \theta_{y} \mathrm{~d} \boldsymbol{\theta} \\ \int \kappa(\boldsymbol{\theta}) \theta_{y} \theta_{x} \mathrm{~d} \boldsymbol{\theta} & \int \kappa(\boldsymbol{\theta}) \theta_{y}^{2} \mathrm{~d} \boldsymbol{\theta}\end{array}\right)$

and its eigenvectors and eigenvalues. The position angle $\phi$ of the lens is given by the angle between one of the eigenvectors of (6) and a unit basis vector. The semimajor and semiminor axes are given by $a=2 \sqrt{\lambda_{1}}$ and $b=2 \sqrt{\lambda_{2}}$ where the $\lambda_{i}$ are the eigenvalues. The ratio $q=b / a$ is a measure of the ellipticity of the mass distribution.

\subsection{Image formation}

The lens equation (4) defines a mapping from $\boldsymbol{\beta}$ to $\boldsymbol{\theta}$. Let $L(\boldsymbol{\theta}, \boldsymbol{\beta})$ denote that mapping. Then, a source-brightness distribution $s(\boldsymbol{\beta})$ will produce an image brightness distribution

$d(\boldsymbol{\theta})=\int L(\boldsymbol{\theta}, \boldsymbol{\beta}) s(\boldsymbol{\beta}) \mathrm{d} \boldsymbol{\beta}$.

This applies if there is perfect resolution on the sky. In general, there will be smearing by the PSF, say $P\left(\boldsymbol{\theta}, \boldsymbol{\theta}^{\prime}\right)$. Hence

$$
\begin{aligned}
d(\boldsymbol{\theta}) & =\int M(\boldsymbol{\theta}, \boldsymbol{\beta}) s(\boldsymbol{\beta}) \mathrm{d} \boldsymbol{\beta} \\
M(\boldsymbol{\theta}, \boldsymbol{\beta}) & =\int P\left(\boldsymbol{\theta}, \boldsymbol{\theta}^{\prime}\right) L\left(\boldsymbol{\theta}^{\prime}, \boldsymbol{\beta}\right) \mathrm{d} \boldsymbol{\theta}^{\prime} .
\end{aligned}
$$

This mapping is linear in the source brightness $s(\boldsymbol{\theta})$, but completely non-linear in the mass distribution. Additionally to the smearing effect by the point-spread function (PSF), the observed image will contain a foreground and noise. Despite the simplicity of equation (8), mass reconstructions of lenses are far more complex. A source brightness can be generated by many different mass distributions. Thus, it is insufficient to optimize mass distributions based on source brightness, and in most cases special information is needed to break those degeneracies.

\section{METHODS}

In this section, we summarize the methodology used in this work. We first describe the SEAGLE pipeline (Mukherjee et al. 2018) and how mock lenses were created (Section 3.1). We then describe the modelling strategy followed in this work with GLASS code (Section 3.2) and summarize the source reconstruction process (in Section 3.3) and comparison with the lens modelling (in Section 3.4).

\subsection{SEAGLE}

SEAGLE is a lens-simulation pipeline based on the EAGLE suite of hydrodynamical simulations of the formation of galaxies and supermassive black holes in a $\Lambda$-cold dark matter universe (Schaye et al. 2014; Crain et al. 2015; McAlpine et al. 2016). EAGLE galaxies are in good agreement with observations of the star formation rate, passive 
fraction, Tully-Fischer relation, total stellar luminosity of galaxy cluster and colours (Trayford et al. 2015), the evolution of the galaxy stellar mass function and sizes (Furlong et al. 2015, 2017), and rotation curves (Schaller et al. 2015). The subgrid physics employed in EAGLE is based on that developed for OWLS (OverWhelmingly Large Simulations Schaye et al. 2010). In this paper, we have chosen to use the reference model L100N1504 which has a maximum proper softening length of $0.7 \mathrm{kpc}$ (see Table 1 in Schaye et al. 2014; Crain et al. 2015).

We apply the SEAGLE pipeline - which incorporates the GLAMER (Metcalf \& Petkova 2014; Petkova et al. 2014) ray-tracing code and the parametric lens-modelling code LENSED (Tessore et al. 2016; Bellagamba et al. 2017) - to selected galaxies from the simulations based on their stellar mass and create their DM, stellar, and gas surface mass density maps with their corresponding lensed images and convergence maps.

The SEAGLE pipeline automatically identifies and extracts samples of (lens) galaxies from the Friends-Of-Friend (FoF) catalogues of the EAGLE simulations. After selecting the galaxy identifiers using an initial selection function, we identify the desired GroupNumber, and SubGroupNumber (numbers assigned to FoF group and subgroup, respectively) and select their particles (gas, DM, and stars). The particles of each galaxy from the simulations are rotated in three different axes and converted into projected surface density maps (or generally referred to as 'mass maps'). The surface density maps are created in units of solar masses per pixel on grids of $512 \times 512$ pixels (for more details of the lensing galaxies, size, and pixel scale of mass maps (see table 2 in Mukherjee et al. 2018). Hereby, the entire galaxy and its local environment are taken into account (see section 3.2 in Mukherjee et al. 2019). They are used as input to the ray-tracing lensing code GLAMER (Metcalf \& Petkova 2014; Petkova et al. 2014). We then choose a source redshift for GLAMER to convert these mass maps into convergence maps. For each convergence map, the critical curves and caustics are calculated to determine where a source has to be placed in order to create multiple-lensed images.

An elliptical Sérsic brightness profile of the source was used with index $n=1$, apparent magnitude $=23$ in the HST-ACS (Advanced Camera for Surveys) F814W filter (AB system) placed at a redshift of $z_{\mathrm{s}}=0.6$ to mimic SLACS (Sloan Lens ACS Survey) lenses. The source has an effective radius of 0.2 arcsec, a position angle $\phi_{s}=0^{\circ}$ and a constant axis ratio $q_{s}=0.6$. The pixel scale $(0.05 \operatorname{arcsec})$, the PSF, and noise correspond to an HST-ACS F $814 \mathrm{~W}$ exposure of typically $2400 \mathrm{~s}$. The final resulting images are exported in standard fits-file format and have sizes of $161 \times 161$ pixels of 8.0 arcsec side length (all parameter values are motivated from SLACS lenses, e. g. Koopmans et al. (2006); Newton et al. (2011)). We randomly choose 15 lensed EAGLE galaxies and their convergence maps (see Figures 2 and 5).

The nomenclature of the lenses depends on their halo, subhalo, and projection catalogue. A number following ' $H$ ' refers to the halo number, 'S' gives the subhalo, and letters 'A/B/G' refers to the projection the galaxy has undergone in Cartesian coordinates i.e. $\alpha, \beta$ and $\gamma$ respectively. Although the names of the lenses have a meaning, it is not important in the context of this paper, and for simplicity we will rather refer to the lenses by their position in the figures.

\subsection{GLASS}

The lens modelling was performed with the free-form modelling code GLASS (Coles et al. 2014). The strategy behind GLASS (and with its precursor PixeLens Saha \& Williams 2004 ) is the following.

First, $\kappa(\boldsymbol{\theta})$ is not a simple parametrized form but a freeform map made up of a few hundred mass tiles or pixels. The arrival time is then

$$
\begin{aligned}
\tau(\boldsymbol{\theta}) & =\frac{1}{2} \theta^{2}-\boldsymbol{\theta} \cdot \boldsymbol{\beta}-2 \sum_{n} \kappa_{n} \psi_{n}(\boldsymbol{\theta}) \\
\psi_{n}(\boldsymbol{\theta}) & =\nabla^{-2} Q_{n}(\boldsymbol{\theta})
\end{aligned}
$$

where $\kappa_{n}$ is the density of the $n$-th mass tile and $Q_{n}(\boldsymbol{\theta})$ represents its shape. Each tile is a square and its contribution $\nabla^{-2} Q_{n}(\boldsymbol{\theta})$ can be worked out exactly (AbdelSalam et al. 1998). The mass tiles are mostly of equal size, but smaller tiles are used in the very central region. We refer to $\sim 200$ tiles as low resolution and $\sim 450$ tiles as high resolution.

Next, point-like features on the images that correspond to a common source are identified using a peak finding algorithm on the lensed images. This provides a set of linear equation for $\kappa_{n}$ and $\boldsymbol{\beta}$. These equations have infinitely many solutions, from which we sample an ensemble of lens models, according to the following priors:

(i) $\kappa_{n} \geq 0$,

(ii) at each image position, the eigenvalues of $\nabla \nabla \tau(\boldsymbol{\theta})$ correspond to minima, saddle points, or maxima in Fermat's principle, as specified for that image,

(iii) each $\kappa_{n}$ is limited to twice the average of its neighbours, to ensure a reasonably smooth distributions,

(iv) the local density gradient is required to point within a specified angle (by default $45^{\circ}$ but usually $80^{\circ}$ in this work) of the centre,

(v) azimuthally averaged density profiles must decrease with increasing radius,

(vi) a constant two-component, external shear is allowed, but each component limited to 0.3 .

Nominal lens and source redshifts of $z_{L}=0.5$ and $z_{S}=$ 3.0 and a concordance cosmology with $\left(\Omega_{m}, \Omega_{\Lambda}, H_{0}^{-1}\right)=$ $(0.3,0.7,14 \mathrm{Gyr})$ were assumed, as this information was unknown during the blind phase. These values do not enter the results however, as the subsequent analysis is entirely in terms of $\kappa$.

\subsection{Source reconstruction and synthetic images}

GLASS treats a source as a point on the source plane that can map on to multiple points on the image plane. Its solutions consist of ensembles of convergence maps which are constrained to reproduce the given image positions from some inferred source position. In reality, we have extended images from extended sources. Extended sources can be emulated by simply using multiple point-like features. For detailed image reconstruction, however, we use a new strategy, an extension of the method used by Küng et al. (2018), involving post-processing the model ensemble from GLASS as follows. 
For each model in the GLASS ensemble, we compute a discrete version $M_{\boldsymbol{\theta} \boldsymbol{\beta}}$ of the lensing and PSF-smearing map $M(\boldsymbol{\theta}, \boldsymbol{\beta})$ in equation (8), by considering $\boldsymbol{\theta}$ values at pixel locations. We then solve for the source-brightness distribution $S_{\boldsymbol{\beta}}$ such that the synthetic image

$D_{\theta}=\sum_{\beta} M_{\theta \beta} S_{\beta}$

best fits the observed brightness $D_{\boldsymbol{\theta}}^{\text {obs }}$ in a least-squares sense. That is to say, we minimize

$\chi^{2}=\sum_{\boldsymbol{\theta}} \frac{\left(\sum_{\boldsymbol{\beta}} M_{\boldsymbol{\theta} \boldsymbol{\beta}} S_{\boldsymbol{\beta}}-D_{\boldsymbol{\theta}}^{\mathrm{obs}}\right)^{2}}{\sigma_{\boldsymbol{\theta}}^{2}}$

for each model in the GLASS ensemble. Once the sourcebrightness distribution has been fitted, it can be reinserted into (10) to generate a synthetic image.

This procedure is formally similar to lens inversion methods previously used by Warren \& Dye (2003); Dye \& Warren (2005); Brewer \& Lewis (2006); Suyu et al. (2006); Vegetti \& Koopmans (2009); Tagore \& Keeton (2014), and many others. In their case, the extended lens images help constrain the source-brightness model and the parametric lens model simultaneously. For such methods, equation (11) is usually supplemented with regularization terms to penalize noisy solutions. A problem which Warren \& Dye (2003) already pointed out is that regularization produces too smooth source-brightness distributions and lead to a bias in the model. Moreover, it was found that mass models are rather insensitive to regularizations if an optimal source pixel size is chosen. Here, the methodology is different. We use equation (11) to solve for synthetic images with already modelled mass maps and thereby test our models' mapping properties. Since we are only interested in the lensed images, regularizations are not necessary.

The uncertainty of an observation corresponding to $\sigma_{\boldsymbol{\theta}}$ in equation (11) generally has several sources, and depends on the optical devices in use. The simplest to model is the photon noise

$\sigma_{\boldsymbol{\theta}}^{2}=g^{-1} D_{\boldsymbol{\theta}}^{\mathrm{obs}}$

where $g$ is the gain or counts per photon. We also assumed a further uniform noise field to mimic other noise sources.

No luminosity and kinematic information about the lensing galaxy was included in the data, nor was any information about the light distribution of the unlensed source. Not having light from the lensing galaxy has one benefit, namely not polluting the lensed image, but also has disadvantage of removing potentially useful information about the lens.

The above is implemented in python so to easily interface with GLASS, but uses optimized parts written in cython (see Behnel et al. 2011) and C. To solve the linear inverse problem it relies on methods provided in the module scipy.sparse and scipy.sparse.linalg (see Jones et al. 2001). Computationally, the most expensive operation is the construction of the sparse matrix $L_{\boldsymbol{\theta} \boldsymbol{\beta}}$ which when multiplied with the PSF yields $M_{\boldsymbol{\theta} \beta}$, because matrix multiplication routines are highly optimized in most frameworks.

\subsection{Model comparison}

Individual models of the lens reconstruction ensemble were compared to the SEAGLE lens models to evaluate their goodness of fit.

First, the resemblance of the convergence maps was investigated. As gravitational lensing is foremost determined by the total mass content of the lens, the ensemble models' Einstein radii - which are a measure of the total mass contained within - were compared to the SEAGLE models. By definition, the scale of the Einstein radius is set where the radial profile of the convergence crosses $\kappa=1$, thus for noncircular lenses we adapt the definition of a notional Einstein radius where $\langle\kappa\rangle_{\mathrm{R}_{\mathrm{E}}}=1$.

Additionally, the shape and orientation of the lens were analysed. The inertia ellipse's semiminor to semimajor axial ratio $q$ acts as a shape parameter and was determined with equation (6). The orientation was determined by the position angle $\phi$ as described in Section 2.3. It can be determined up to an ambiguity of $\pm \pi$, provided the lens is not perfectly round (which happens in the limit of $q \rightarrow 1$ ). To combat this degeneracy, we define a complex ellipticity

$\epsilon=\frac{1-q}{1+q} e^{2 i \phi}$

which combines the ellipticity and the position angle following Mukherjee et al. (2018). With this definition, round lenses will fall closer to the origin no matter what position angle, whereas more elliptical lenses move away from the origin at an angle given by the position angle.

As mentioned before, the relation between convergence and synthetic image is degenerate. This means, if the convergence exactly matches the 'true' convergence, the synthetic image and the observed data will be indistinguishable. Slight changes in the convergence however do not linearly translate to the synthetic image, and vice versa. A straight-forward comparison of the model's convergence to the 'true' convergence map is thus insufficient. We therefore use the Roche potential as in equation (5) as a basis of comparison. The best-fitting model in the ensemble can be found with the maximum modulus of the inner product of the modelled lensing Roche potential $\mathcal{P}^{\text {mod }}$ and the lensing Roche potential of the original convergence map $\mathcal{P}^{\text {orig }}$.

$\max \left\langle P^{\text {orig }}, P^{\text {mod }}\right\rangle=\max \left|\frac{\int \mathcal{P}^{\text {orig }}(\boldsymbol{\theta}) \mathcal{P}^{\text {mod }}(\boldsymbol{\theta}) \mathrm{d} \boldsymbol{\theta}}{\sqrt{\int\left|\mathcal{P}^{\text {orig }}\right|^{2} \mathrm{~d} \boldsymbol{\theta}} \sqrt{\int\left|\mathcal{P}^{\text {mod }}\right|^{2} \mathrm{~d} \boldsymbol{\theta}}}\right|$

Since the Roche potentials are independent of the source position and the inner product is normalized, the mass-sheet degeneracy is completely eliminated. The masssheet degeneracy not only affects a mass distribution's overall amplitude by adding (or subtracting) an arbitrary number of mass sheets of critical density, but more importantly also changes its scale and steepness. This means, even though a model's convergence profile might not show perfect match in its slope, it can very well be a valid solution.

\section{RESULTS}

The sample of 15 SEAGLE lenses is described in Section 3.1 and shown in Figure 2. These lensed images were prepared 
by SM. PD, in consultation with JC and PS, reconstructed the lenses from these data. Besides the mass centroid of the lensing galaxy and the PSF used to blur the lensed images, no other information about the lenses was provided to the modellers.

The lens reconstructions were done in two phases. In the first phase, three lenses were unblinded early, in order to test and improve the pipeline. In the second phase, PD modelled the other 12 lenses unaware of the truth held by SM.

The lenses in the top row were chosen for the first phase because of their diverse lensed image configuration. As shown in Figure 2, the lens on the left is a quad lens with an almost ring-like image configuration, which is present in most regular quads and doubles in the sample, the lens in the middle is a double with a wide arc image and a less extended image slightly closer to the lens, and the lens on the right is one of five systems presenting a fifth non-demagnified image, which is always a maximum of the arrival time. This subset covers the most typical lens properties in the entire sample (from a lens modeller's perspective). After the comparison of the three lens models with their actual convergence, the lens-modelling procedure was adjusted to be better prepared for the second phase.

In the second phase, the remaining lenses were reconstructed with the actual convergence maps veiled. The latter were then revealed for comparison, but no further changes to the models were allowed.

It is important to note that our goal during the lens reconstruction was not simply to optimally reproduce the lenses images, but to find an ensemble sampling the possible mass maps.

\subsection{Lens reconstructions}

The lens modelling directly yields ensembles of $\kappa$ maps for the lenses. Synthetic images for each mass map in the ensemble were constructed and a $\chi^{2}$ was evaluated for each image according to equation (11). In each ensemble at least half of all synthetic images gave bad fits to the image data, however for 12 of the 15 lenses there was at least one synthetic image which fit the data well. Figure 3 shows the synthetic image with the minimal $\chi^{2}$ in the ensemble, for each lens. As described in Section 3.3, the synthetic images were constructed by projecting the data from the image plane on to the source plane using the model to calculate the deflection angles, and reprojecting back on to the image plane. Interestingly, and against our initial expectations, low-resolution models often seem to produce better synthetic images. The reduced $\chi^{2}$ values never get to 1 , indicating that the best fits are dominated by systematic errors in the model fitting. The high-resolution images have reduced $\chi^{2}$ of $\sim 2.5$, whereas the low-resolution images have values as low as 1.7.

Although it is sometimes possible to spot irregularities and unphysical features in the mass maps from simple inspection, it is generally easier to review the contours of the derived arrival-time surface (9). Arrival-time surfaces become very intuitive to interpret once the saddle-point contours have been drawn, as they already schematically resemble lensed arcs and can visually be compared to the image data. Figure 4 shows arrival-time surfaces of the ensembleaverage models of each lens. Saddle-point contours are in- dicated in black, and image position constraints for minima, saddle-points, and maxima in blue, purple, and red respectively. The models by construction reproduce point-like image features at the correct positions. But they can also show additional spurious images, easily identifiable as local extrema. Ensemble averages tend to suppress these spurious images. The arrival-time surfaces from GLASS are highly sensitive to the image positions. In our experience, good models produce nice smooth-looking arrival-time surface, but so do some bad models; whereas ragged-looking arrivaltime surfaces invariable indicate bad models. Slight shifts in the image positions might result in significantly different arrival-time surfaces and mass maps. The fact that the lenses have extended, and sometimes almost ring-shaped images, aggravates this difficulty. As the time delays were unknown, the parity of the extrema was uncertain as well. In some cases, a trial-and-error approach had to be adopted until a credible model was obtained, with others an educated guess could be made based on the distances of the images to the lens and their brightness.

During the reconstructions, the prior parameters in GLASS were tweaked to obtain a satisfactory arrival-time surface of the ensemble average in each case. Additionally, we filtered out a percentage of the worst $\kappa$ maps from the distribution of $\chi^{2}$, and by mere construction improved the ensemble average's synthetic image. This gave considerable improvements for ensembles with a wide variety in its models, however only slight changes for ensembles with a less diverse set.

\subsection{Convergence map comparison}

The $\kappa$ maps used by SM to generate the lenses are shown in Figure 5. Apart from the top row, these were hidden during the modelling process. The modelling generated ensembles of $\kappa$ maps, and the ensemble averages are shown in Figure 6. The modelled convergence maps show the mass-tile structure of the free-form method, whereas the actual maps have higher pixel resolution. A direct comparison of the convergences was expected to be insufficient due to the well-known problem of degeneracies. The numerically best-fitting convergence map of an ensemble according to

$\min _{\text {model }} \sum_{i}\left(\kappa_{i}^{\text {truth }}-\kappa_{i}^{\text {model }}\right)^{2}$

produced bad or mediocre synthetic images in all lens models, which further confirmed our suspicion. We visually inspected all ensembles each with 1000 individual models. In some ensembles, the ellipticities and position angles had little spread and were definitive, but in others, the models showed an ambiguity of $\pm \pi$ in their position angles. We therefore compared the radial profiles and ellipticities, as follows.

Figure 7 shows the average enclosed $\kappa$ as a function of radial distance from lens centre. The formal Einstein $\mathrm{R}_{\mathrm{E}}$ corresponds to $\langle\kappa\rangle_{\mathrm{R}_{\mathrm{E}}}=1$. The Einstein radii are well recovered and have a median relative error of 4.3 per cent over the entire set of quads; for some quads, the Einstein radius had an error as low as 1.0 per cent, and was slightly overestimated for others with maximally 15.7 per cent, but in all cases the errors were smaller than the pixel sizes. The Einstein radii for double systems were less accurate with an average error 
of 24.8 per cent. But even so, the profiles are systematically too shallow. It is interesting, however, that the model profiles (green-yellow bands) could be brought much closer to the correct profiles (red curves) by multiplying $1-\kappa$ by a constant. This transformation is nothing but the steepness degeneracy, which does not affect the images.

Figure 8 compares the complex ellipticity (13) for the model ensembles with the SEAGLE values. Without exception, the lens models tend to be too round. This makes it more difficult to determine a position angle for the models. Nevertheless, in all but two cases the position angle was roughly recovered with a median error of $\pm 9.4^{\circ}$. This is also observable by comparing corresponding maps in Figure 5 and Figure 6.

The problematic lenses are in the bottom row, especially the two in the middle and right-hand panels. In both cases, the position angles are off by almost $90^{\circ}$. This is evident in Figure 8 as the ellipticity of the ensemble model is in the quadrant opposite to the one of the SEAGLE simulation. This is also observable by directly comparing the orientation of the galaxies in Figure 5 and Figure 6. After comparing the arrival-time surfaces and convergences, we suspect the image order is most likely wrong. In both cases, the arc to the north should have been diagnosed as arriving before the counterimage to the south, whereas in the models the opposite was done. The estimated semimajor and semiminor axes which appear in the complex ellipticity only as a ratio, also were generally too high compared to the SEAGLE simulations. This indicates that the lens ensemble models have more mass at higher radii from the centre relative to the total mass, as the semimajor and semiminor axes were estimated with the inertia ellipses of the convergences. This means, as we have already seen, that the models tend to be too shallow across the board.

\subsection{Roche potential comparison}

After unblinding the actual convergence maps of the lenses in the top row, it became clear that a further comparison was desirable which quantified the goodness of the lens reconstruction with the effect of the steepness degeneracy taken out. We then formulated and calculated the lensing Roche potential. This is just the arrival time from equation (9) without the $\boldsymbol{\theta} \cdot \boldsymbol{\beta}$ term and an arbitrary additive constant. Furthermore, the effect of the steepness degeneracy corresponds to an arbitrary multiplicative factor, as explained in Section 2.2. Thus, we are free to subtract the potential's value at the centre from the entire map and to normalize to the negative of its minimal value. This way the lowest minimum has the value -1 and the map centre the value 0 , and takes out the effect of the steepness degeneracy. The results from the actual convergence maps and from the lens models are shown in Figure 9 and Figure 10 respectively. These figures show contoured regions of equal level of the Roche potentials as in equation (5).

In a rough visual comparison, most lens models - except the already mentioned lenses in the bottom row show the same main features and morphologically appear to agree with their SEAGLE counterpart. In particular, the position and shape of minima and the lens' orientation match quite well. For some quad systems such as the left one in the second-last row, there seems to be a tilt in the model which is suppressing one of the minima, which is not evident in the corresponding SEAGLE simulation. Similarly, some saddle regions of the models seem to have switched amplitudes causing lemniscates to wrap around the central maximum from a different side compared to the SEAGLE simulations.

A quantitative comparison of the Roche potentials was done by evaluating the scalar product (14). The black circles in Figure 9 and 10 indicate the radii within which integrals in the scalar product were evaluated. It was necessary to choose a radius smaller than the map radius as otherwise the scalar product would have been dominated by differences on the edges of the image plane. Using a small integration radius instead, we observed that differences in the shape of the central region and regions near the saddle-contours of the potential dominate the scalar product.

Filtering out models from the ensemble which give low scalar product values will by construction yield an overall improved ensemble average, since the fraction of bad models shrinks.

It is now interesting to put in contrast the synthetic image and the Roche potential as criteria. This means comparing the distribution of $\chi^{2}$ from the synthetic images and the distribution of scalar products of the Roche potentials $\left\langle P^{\text {orig }}, P^{\text {mod }}\right\rangle$ within an ensemble. This comparison is illustrated by the hex-binned plots in Figure 11. Just as we expect a filtering of the ensemble in which only models with low $\chi^{2}$ of the synthetic image are kept to improve the ensemble overall, filtering according to the Roche potential is expected to elevate the quality of ensembles as well. Hence we expect both methods to anticorrelate, meaning, models with a low $\chi^{2}$ should to have a scalar product of the Roche potential with a value close to 1 , whereas higher $\chi^{2}$ should have a low scalar product. At first glance, Figure 11 neither disproves this assumption, nor conclusively confirms it. None of the lenses clearly display tendencies towards anticorrelation between $\chi^{2}$ and $\left\langle P^{\text {orig }}, P^{\text {mod }}\right\rangle$. However, it seems that mostly models with a diverse ensemble show an anticorrelative trend, whereas models with an already overall high-quality ensemble distribute rather uniformly in that parameter space. It is also interesting that some lenses, e.g. the lens in the middle row to the right (H30S0A0B90G0), show a wide spread in the scalar product of the Roche potentials, but a very low spread in $\chi^{2}$. This means that even though the shapes of the mass maps differ widely, the mass maps produce similar quality of synthetic images. This implies that filtering should improve broad ensemble models, however there is a point when optimizing an ensemble model does not change the model anymore, at least globally. This is probably due to the fact that those models seem to agree on the global structure of the convergence and continue fitting substructures.

An example of this is also shown Figure 12. The differences are minor, but reducing the ensemble to only 100 models with the lowest $\chi^{2}$ visibly improves the synthetic image of the ensemble-averaged model, which was expected as the $\chi^{2}$ is a direct measure of the synthetic images. However, trying to improve that ensemble again by filtering out the worst scalar products of the Roche potentials does not clearly improve upon the already filtered ensemble anymore. This can be explained by the fact that in the end synthetic images are a local, non-linear measure of the mass whereas 
the Roche potential is a direct measure of the global mass model.

\section{CONCLUSION}

We need to prepare for the tens of thousands of strong gravitational lenses expected to be discovered with the next generation of wide-field telescopes: Euclid and WFIRST (WideField Infrared Survey Telescope) in space and LSST (Large Synoptic Survey Telescope) on the ground. Lately, many efforts have been made to prepare for this data flood, from automatic identification of lens candidates with the use of machine learning, to automatic modelling of lenses with novel codes. While this paper does not address the problem of scalability for future big data sets, it demonstrates the necessity of such blind test, particularly for lens-modelling tools which were designed to scale up to thousands of lenses. It is still unclear how much we can trust the resulting lens models of such pipelines and what aspects of a lens model are robust against degeneracies. The only way to objectively test lens reconstruction techniques and avoid confirmation bias are blind tests with realistically simulated data.

In this work, we have used a sample of 15 simulated strong lenses from the state-of-the-art hydrodynamical simulation of EAGLE and modelled them blind with the GLASS code. We introduced a new lensing potential, the 'Roche potential' and showed that using this in free-form modelling we are successfully able to reconstruct the lensing systems without the mass-sheet or steepness degeneracy.

General properties like extended image information, Einstein radii, and shape of the convergence have been investigated and compared to the actual, subsequently unveiled convergence. Thereby, the following results stood out:

- It was straightforward to find models in the ensemble with good fitting synthetic images. Low-resolution models actually tended to perform better in this respect. It appears that high-resolution models start fitting substructures which predominantly impact the synthetic images. Also, low-resolution images produce smoother source images, which might also improve fits to the images. This can be seen when comparing Figure 1 which shows synthetic images constructed with low-resolution models and Figure 3 which displays the image reconstructions from high-resolution models.

- The Einstein radii were recovered quite well for most lenses, in some cases they were slightly overestimated.

- The position angles were also approximately in agreement, except for two lenses. In those two cases, the problems were traced back to having chosen the wrong image order in the lens reconstruction.

- The ellipticities of the mass maps were generally too round.

- The radially averaged convergence profiles were all found to be too shallow. However, this is an effect of the steepness degeneracy and could be resolved by multiplying the $1-\kappa$ surface by an arbitrary factor.

Even when all those properties are approximately recovered by a lens model, it was demonstrated that this does not necessarily mean the actual distribution has been found.

The reason why reconstructing lenses is so difficult lies in the many kinds of degeneracies that affect lens models. The most important of these is the steepness degeneracy (also called the mass-sheet degeneracy). The novel concept of a lensing Roche potential is introduced to remove the effect of the steepness degeneracy, that is, to compare models which have been differently affected by the steepness degeneracy. The scalar product of two Roche potentials gives a true measure of how alike models are to each other whilst ignoring the steepness degeneracy. A good match between model and truth simply means that up to an arbitrary rescaling of $1-\kappa$ the shape of $\kappa$ agrees well with the truth. In the cases where the scalar product of the Roche potentials is far from 1 , we have a bad match, meaning the actual shape of $\kappa$ has not been recovered well enough.

We demonstrated that filtering out models where the synthetic images have the highest $\chi^{2}$ seems to improve ensembles up to a point. However, once the global properties of the lens have been modelled, further optimization of the synthetic images does not improve fits to substructures and other local properties. This means, even if synthetic images from a model ensemble might show only minor variation, the underlying convergence might have major differences in comparison. This shows that the scalar product of Roche potentials is a better criterion to determine a model's quality when mass distributions are of primary interest.

In summary, this study not only confirms the wellknown characteristic of lens modelling, that the Einstein radius of the mass distribution is generally well reproduced whereas its steepness is not, but also highlights additional points:

- To mitigate the problem of unbroken degeneracies, we recommend producing an ensemble of many solutions rather than a single model.

- While synthetic images represent a convenient visual diagnostic, they are useful only up to a point. Once a synthetic image is reasonably good (and well before the formal $\chi^{2}$ criterion is achieved) the goodness of the synthetic image does not correlate with the goodness of the lens model. In other words, even if the model reproduces lensed images which resemble the data rather nicely, the mass map might still be far from the truth.

- Careful attention should be paid to the parity and time order of the images, because getting these incorrect, results in poor models.

- When testing lens-modelling tools against well-known lenses or simulations, the scalar product of the Roche potentials gives a measure of the shape similarity of the mass models which is robust against degeneracies. If this scalar product indicates a bad match, a recalibration of the lensmodelling tool might be needed.

\section{FUNDING STATEMENT}

PD acknowledges support from the Swiss National Science Foundation. SM acknowledges the funding from the European Research Council (ERC) under the EUs Horizon 2020 research and innovation programme (COSMICLENS; grant agreement no. 787886). 


\section{REFERENCES}

AbdelSalam H. M., Saha P., Williams L. L. R., 1998, MNRAS, 294,734

Auger M. W., Treu T., Bolton A. S., Gavazzi R., Koopmans L. V. E., Marshall P. J., Moustakas L. A., Burles S., 2010, ApJ, 724,511

Barnabè M., Czoske O., Koopmans L. V. E., Treu T., Bolton A. S., 2011, MNRAS, 415, 2215

Behnel S., Bradshaw R., Citro C., Dalcin L., Seljebotn D. S., Smith K., 2011, Computing in Science \& Engineering, 13, 31

Bellagamba F., Tessore N., Metcalf R. B., 2017, MNRAS, 464, 4823

Blandford R., Narayan R., 1986, ApJ, 310, 568

Brewer B. J., Lewis G. F., 2006, ApJ, 651, 8

Coles J. P., Read J. I., Saha P., 2014, MNRAS, 445, 2181

Courbin F., Saha P., Schechter P. L., 2002, Quasar Lensing. Springer Berlin Heidelberg, Berlin, Heidelberg, pp 1-54, doi: $10.1007 / 3-540-45857-3^{*} 1$

Crain R. A., et al., 2015, MNRAS, 450, 1937

Dye S., Warren S. J., 2005, ApJ, 623, 31

Furlong M., et al., 2015, MNRAS, 450, 4486

Furlong M., et al., 2017, MNRAS, 465, 722

Hezaveh Y. D., Levasseur L. P., Marshall P. J., 2017, Nature, 548, 555

Hutsemékers D., Braibant L., Sluse D., Goosmann R., 2019, A\&A, 629, A43

Jacobs C., Glazebrook K., Collett T., More A., McCarthy C., 2017, MNRAS, 471, 167

Jones E., Oliphant T., Peterson P., et al., 2001, SciPy: Open source scientific tools for Python, http://www.scipy.org/

Koopmans L. V. E., Treu T., 2003, ApJ, 583, 606

Koopmans L. V. E., Treu T., Bolton A. S., Burles S., Moustakas L. A., 2006, ApJ, 649, 599

Koopmans L. V. E., et al., 2009, ApJ, 703, L51

Küng R., 2018, Astronomy and Computing, 23, 115

Küng R., et al., 2015, MNRAS, 447, 2170

Küng R., et al., 2018, MNRAS, 474, 3700

Lanusse F., Ma Q., Li N., Collett T. E., Li C.-L., Ravanbakhsh S., Mandelbaum R., Poczos B., 2017, MNRAS, 473, 3895

Leier D., Ferreras I., Saha P., Falco E. E., 2011, ApJ, 740, 97

Leier D., Ferreras I., Saha P., Charlot S., Bruzual G., La Barbera F., 2016, MNRAS, 459, 3677

Lubini M., Coles J., 2012, MNRAS, 425, 3077

Marshall P. J., et al., 2016, MNRAS, 455, 1171

McAlpine S., et al., 2016, Astronomy and Computing, 15, 72

Meneghetti M., et al., 2017, MNRAS, 472, 3177

Metcalf R. B., Petkova M., 2014, MNRAS, 445, 1942

More A., et al., 2016, MNRAS, 455, 1191

Mukherjee S., et al., 2018, MNRAS, 479, 4108

Mukherjee S., Koopmans L. V. E., Metcalf R. B., Tortora C., Schaller M., Schaye J., Vernardos G., Bellagamba F., 2019, arXiv e-prints,

Newton E. R., Marshall P. J., Treu T., Auger M. W., Gavazzi R., Bolton A. S., Koopmans L. V. E., Moustakas L. A., 2011, ApJ, 734, 104

Nierenberg A. M., et al., 2017, MNRAS, 471, 2224

Nightingale J. W., Dye S., Massey R. J., 2018, MNRAS, 478, 4738

Oguri M., Marshall P. J., 2010, MNRAS, 405, 2579

Paraficz D., Hjorth J., 2010, ApJ, 712, 1378

Petkova M., Metcalf R. B., Giocoli C., 2014, MNRAS, 445, 1954

Read J. I., Saha P., Macciò A. V., 2007, ApJ, 667, 645

Saha P., 2000, AJ, 120, 1654

Saha P., Williams L. L. R., 1997, MNRAS, 292, 148

Saha P., Williams L. L. R., 2004, AJ, 127, 2604

Saha P., Williams L. L. R., 2006, ApJ, 653, 936

Schaller M., et al., 2015, MNRAS, 451, 1247

Schaye J., et al., 2010, MNRAS, 402, 1536
Schaye J., et al., 2014, MNRAS, 446, 521

Schneider P., Sluse D., 2014, A\&A, 564, A103

Sereno M., Paraficz D., 2014, MNRAS, 437, 600

Sluse D., Hutsemékers D., Courbin F., Meylan G., Wambsganss J., 2012, A\&A, 544, A62

Suyu S. H., Halkola A., 2010, A\&A, 524, A94

Suyu S. H., Marshall P. J., Hobson M. P., Blandford R. D., 2006, MNRAS, 371, 983

Suyu S. H., et al., 2017, MNRAS, 468, 2590

Tagore A. S., Keeton C. R., 2014, MNRAS, 445, 694

Tessore N., Bellagamba F., Metcalf R. B., 2016, MNRAS, 463, 3115

Tomozeiu M., Mohammed I., Rabold M., Saha P., Wambsganss J., 2018, MNRAS, 475, 1925

Trayford J. W., et al., 2015, MNRAS, 452, 2879

Treu T., Koopmans L. V. E., 2004, ApJ, 611, 739

Vegetti S., Koopmans L. V. E., 2009, MNRAS, 392, 945

Walsh D., Carswell R. F., Weymann R. J., 1979, Nature, 279, 381

Warren S. J., Dye S., 2003, ApJ, 590, 673

Williams L. L. R., Saha P., 2000, AJ, 119, 439

Young P., Deverill R. S., Gunn J. E., Westphal J. A., Kristian J., 1981, ApJ, 244, 723

\section{FIGURES}

This section contains all figures which are referenced in previous sections. 

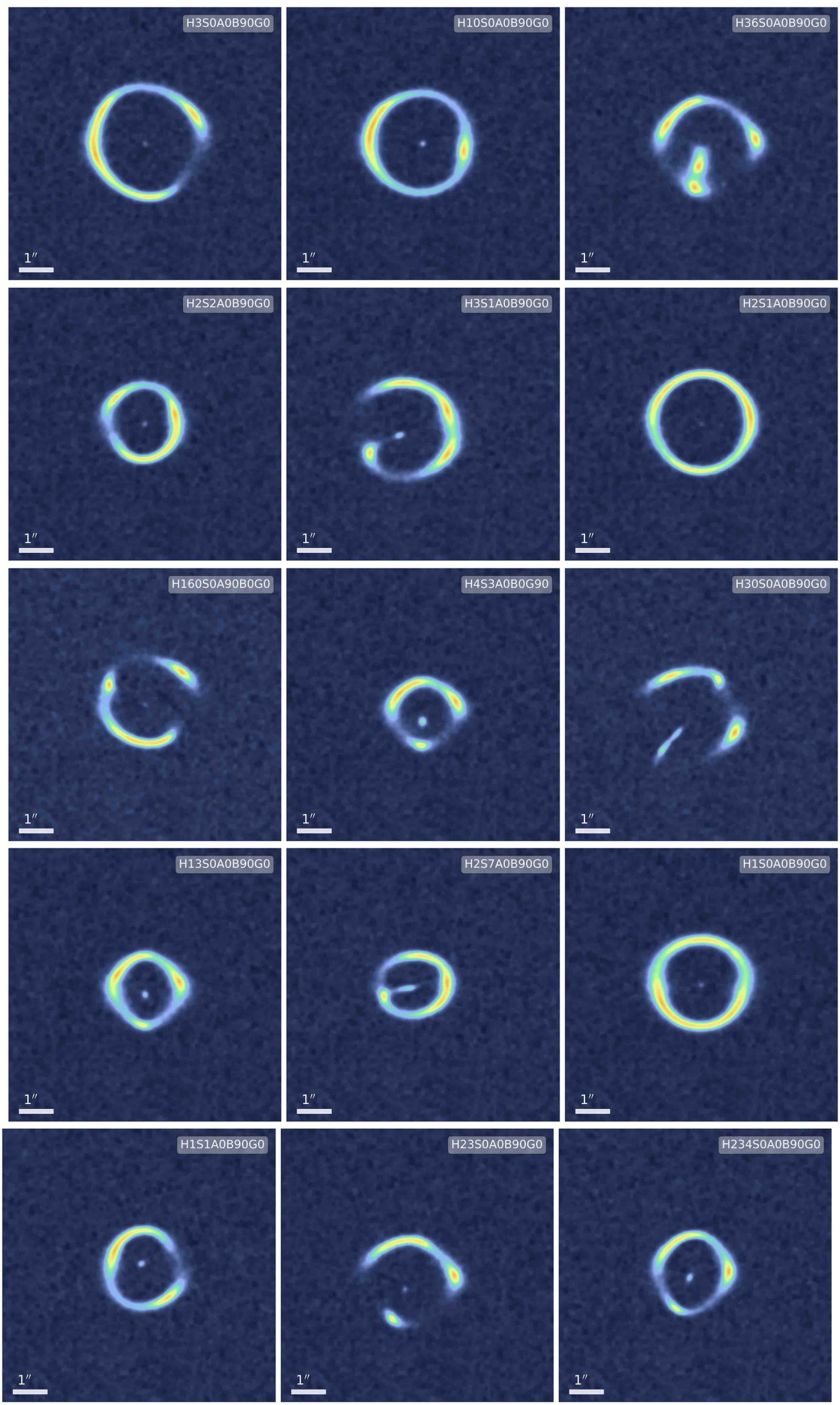

Figure 2. The SEAGLE-simulated lens data. The pictures show the lensed images without the lens in arbitrary units of brightness. They were the only information provided in the blind study. The scale bar on the lower left in each panel shows the physical scale in arcseconds. 


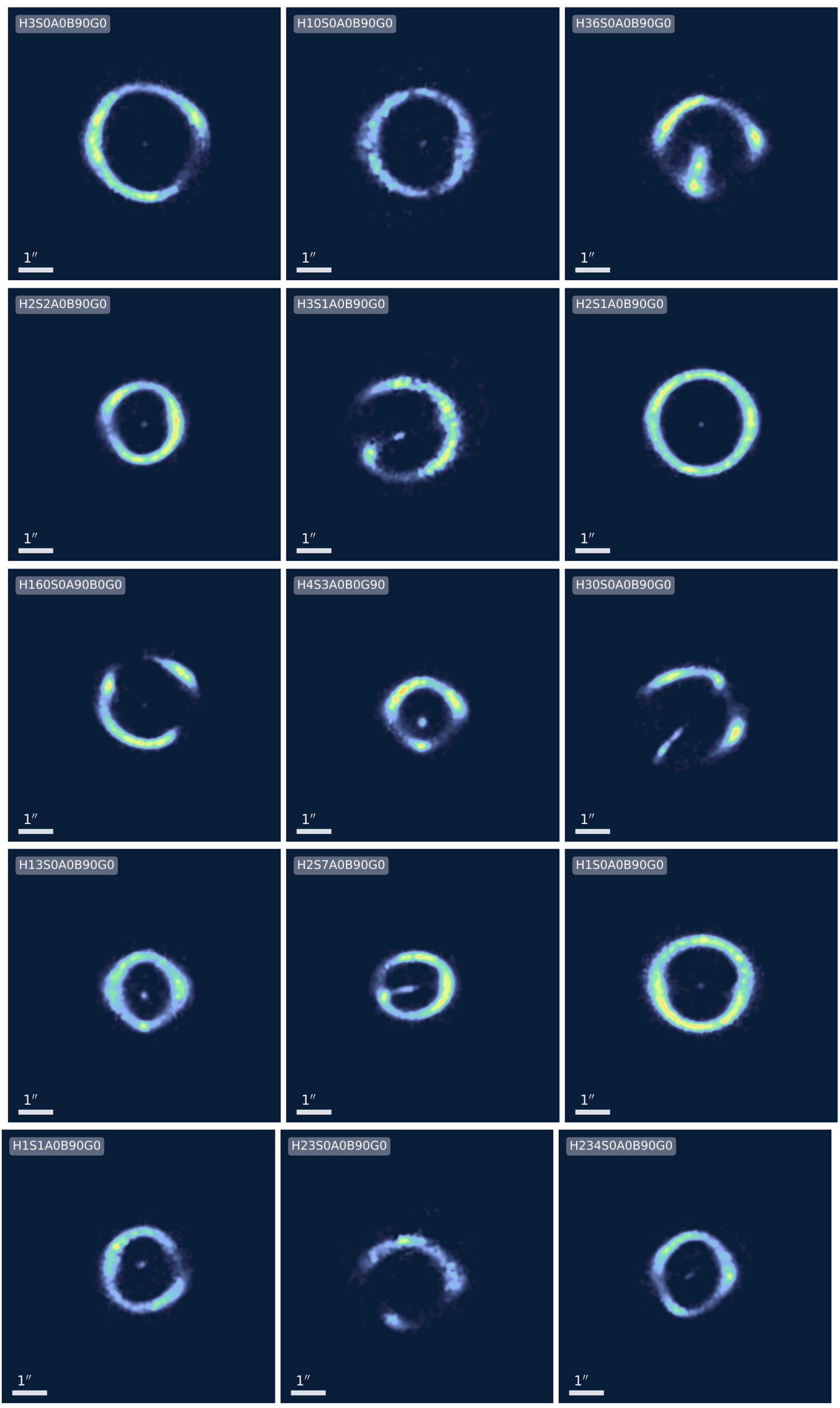

Figure 3. Synthetic images of the 15 reconstructed lenses using the best-fitting model from the ensemble solution. Scales are identical to the corresponding pictures in Figure 2. 
Denzel et al.

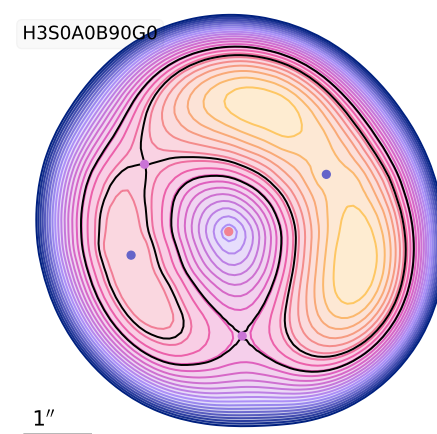$$
1^{\prime \prime}
$$
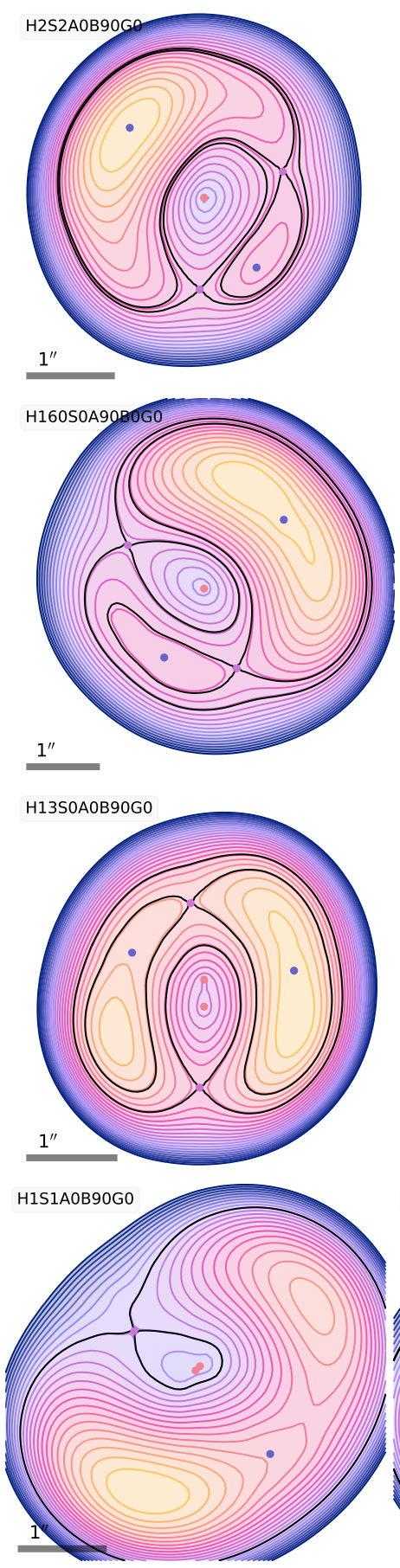
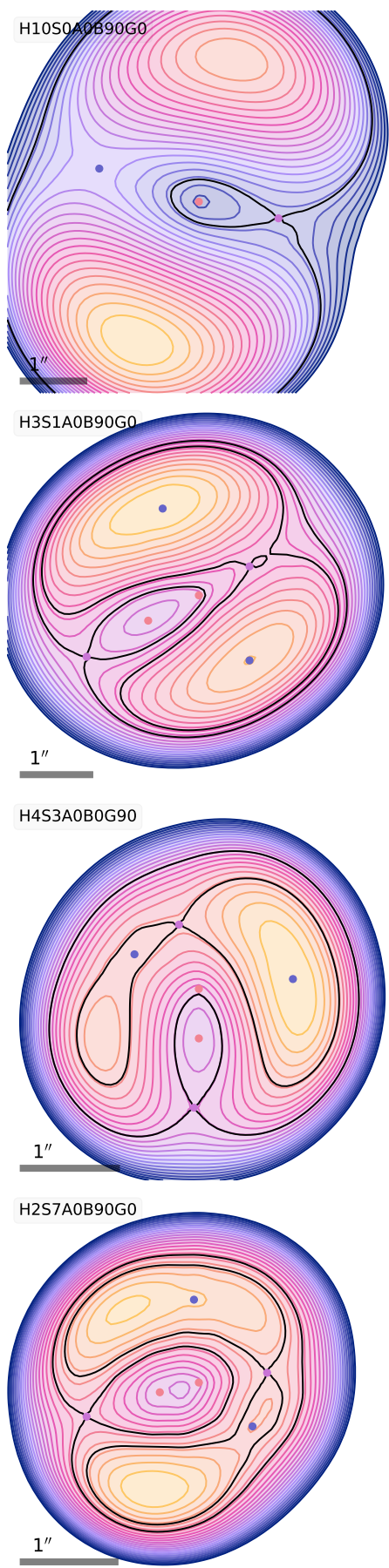

H23SOAOB9OGO

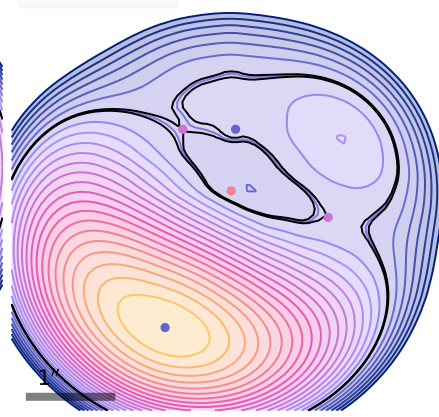

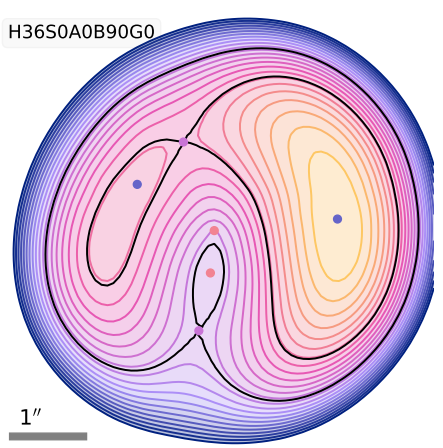

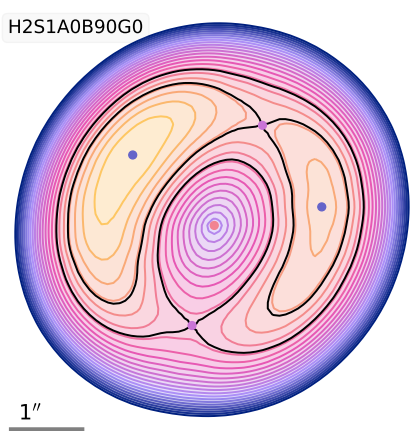

H3OSOAOB9OGO
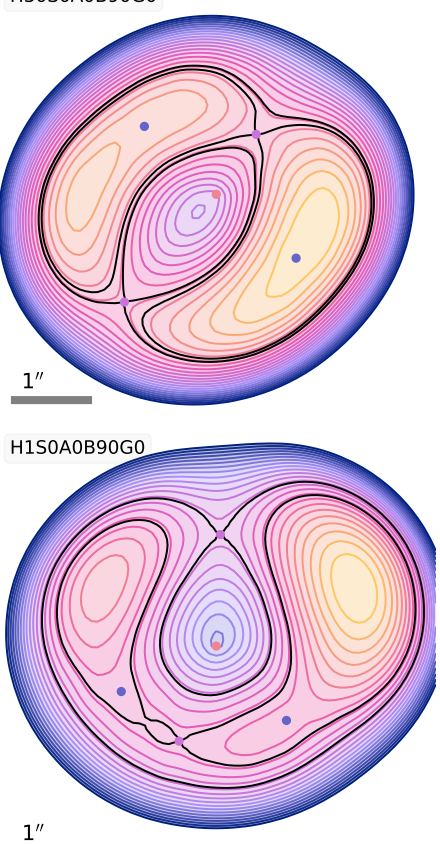

$1^{\prime \prime}$

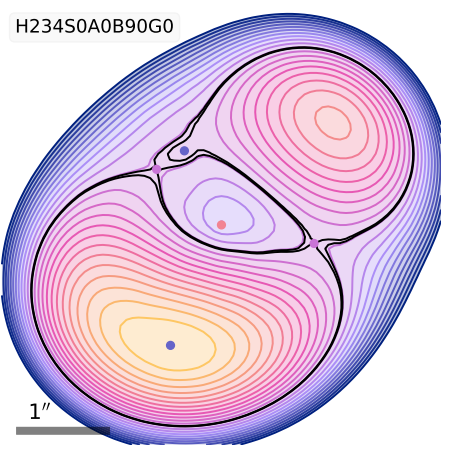

Figure 4. Arrival-time surfaces of the ensemble average of the 15 reconstructed lenses. Contours passing through saddle points are in black. The lens centre is indicated by a red dot, while the image-position constraints with minimum, saddle, and maximum parity are indicated by blue, purple, and red dots respectively. It is easy to identify unrealistic models by looking for irregularities in the arrival-time surface contours. 


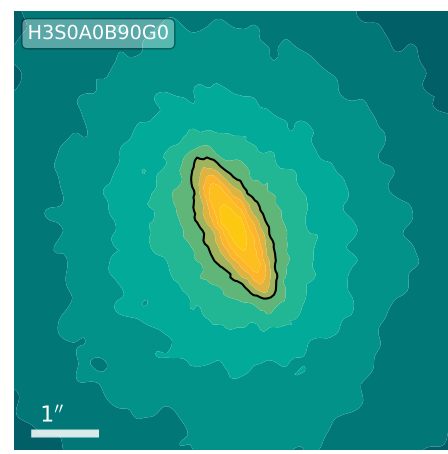

H2S2AOB90GO


H13SOAOB90G0

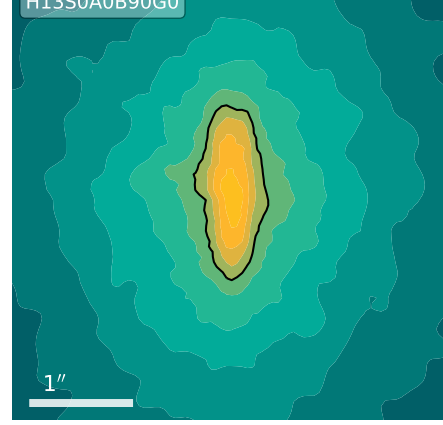

H1S1A0B90G0

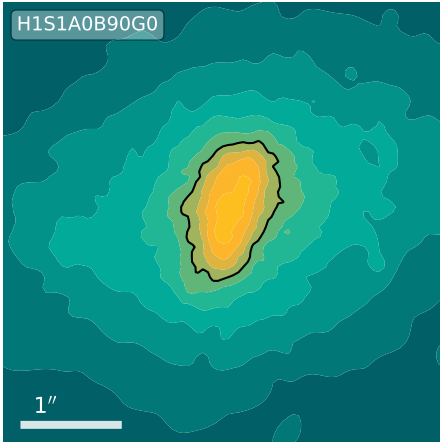

H1OSOAOB9OGO

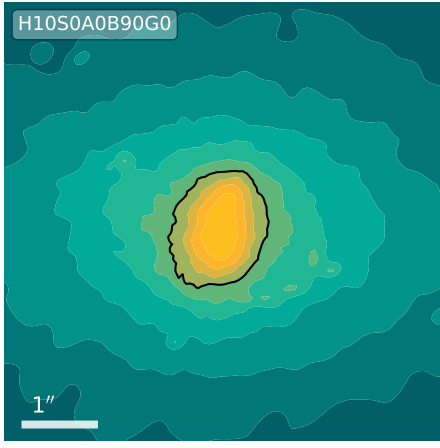

H3S1AOB $90 \mathrm{GO}$

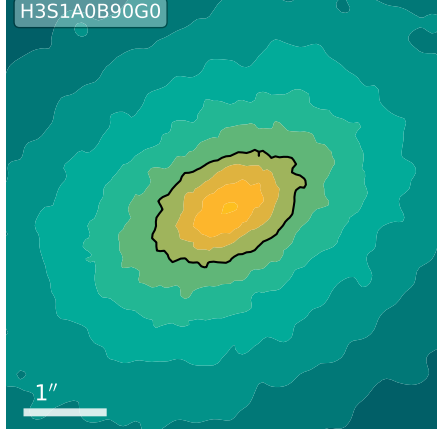

H4S3A0B0G90

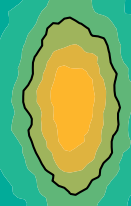

$1^{\prime \prime}$

H2S7A0B90G0

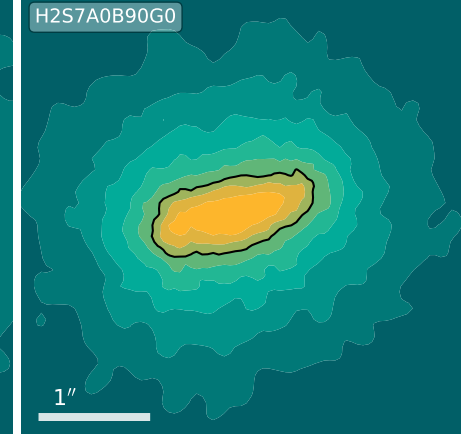

H23SOAOB $90 G 0$

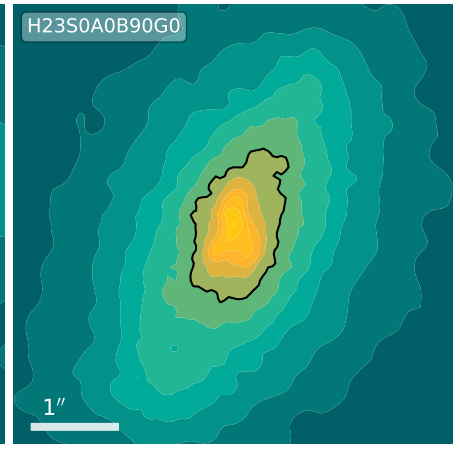

H36SOAOB90GO

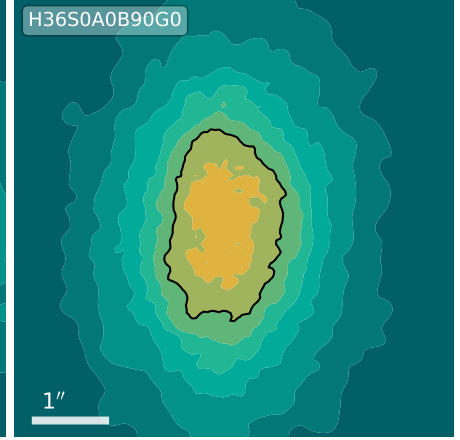

H2S1AOB90GO
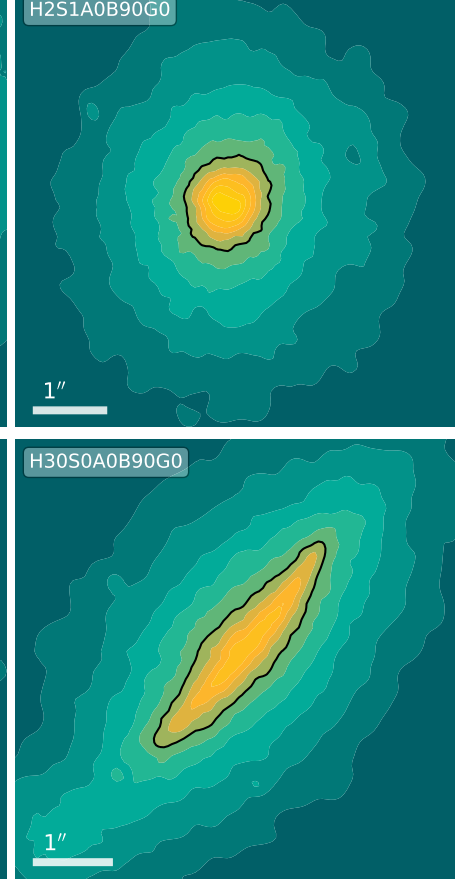

H1SOAOB90GO

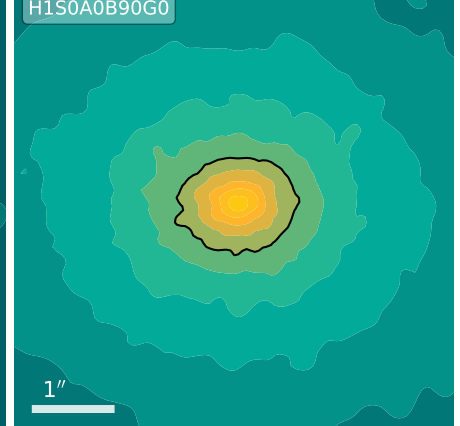

H234SOAOB90GO

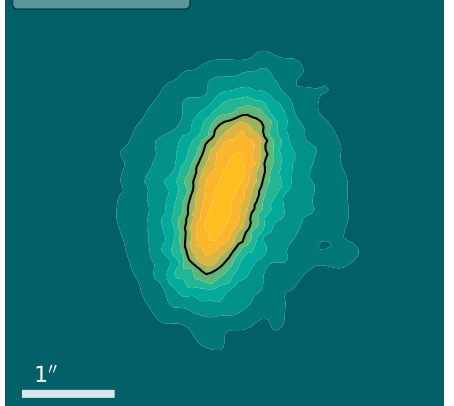

Figure 5. The actual convergence maps of SEAGLE-simulated lenses. Black contours indicate a $\kappa=1$. They were unblinded after the lens reconstructions were completed. 
Denzel et al.
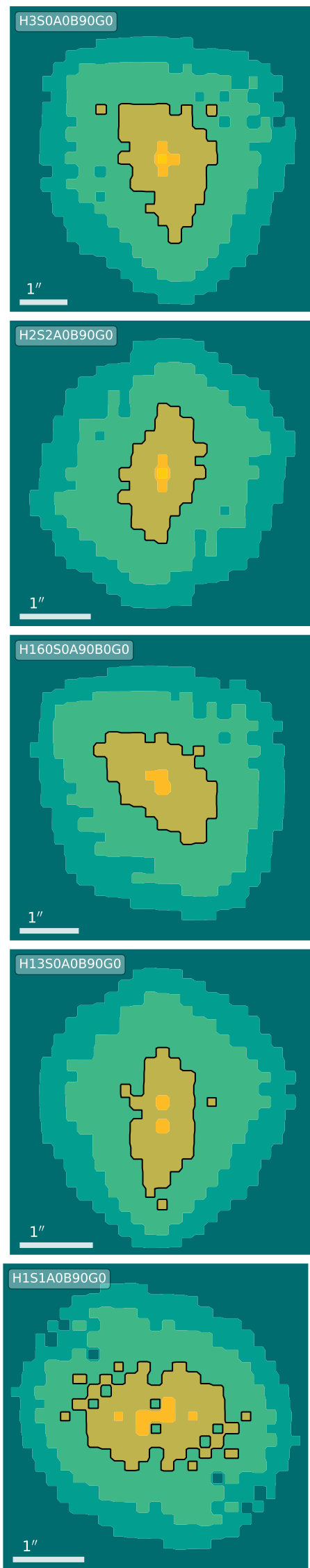
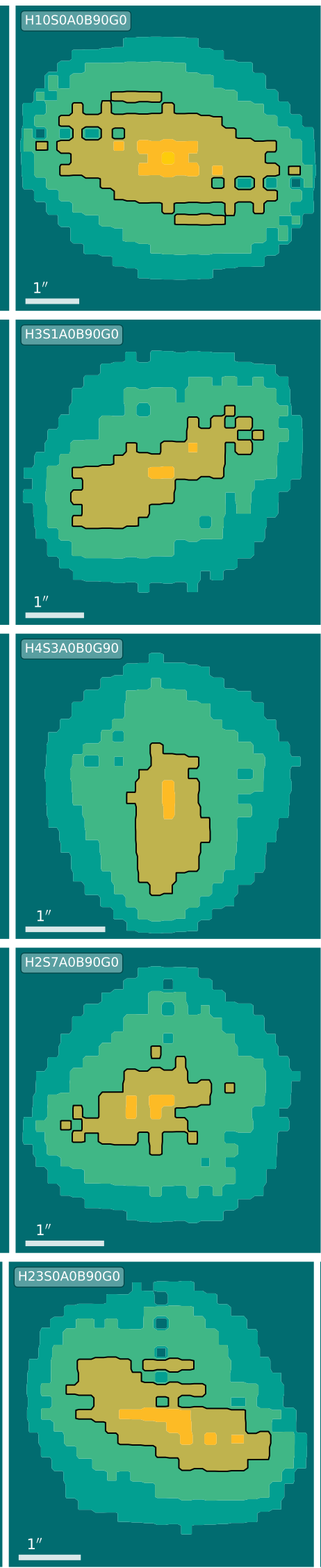
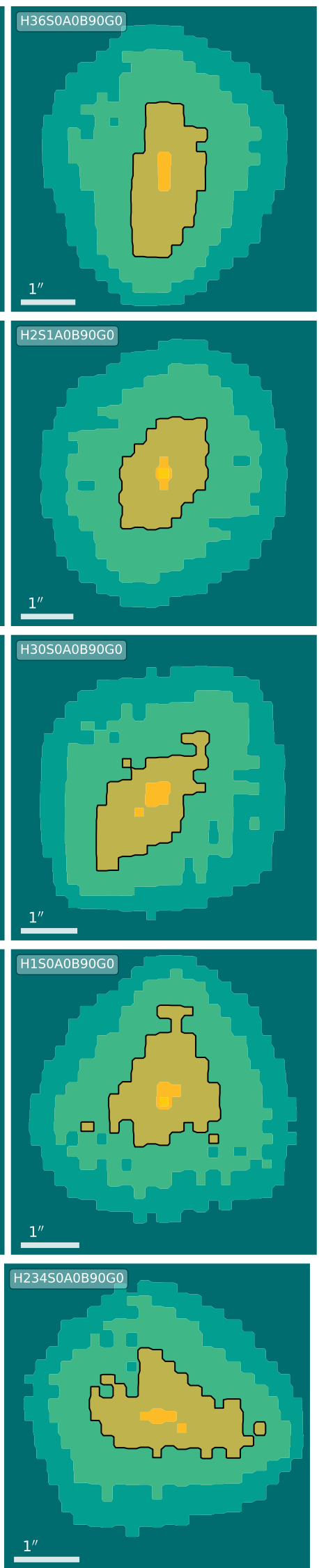

Figure 6. Model convergence maps (ensemble-averages) of all the lenses. Black contours indicate a $\kappa=1$. Scales are identical to the corresponding pictures in Figure 5. Direct comparison of convergence maps usually fails, because they are affected by degeneracies. Nevertheless, the orientation of the lenses roughly match their SEAGLE counterparts. 


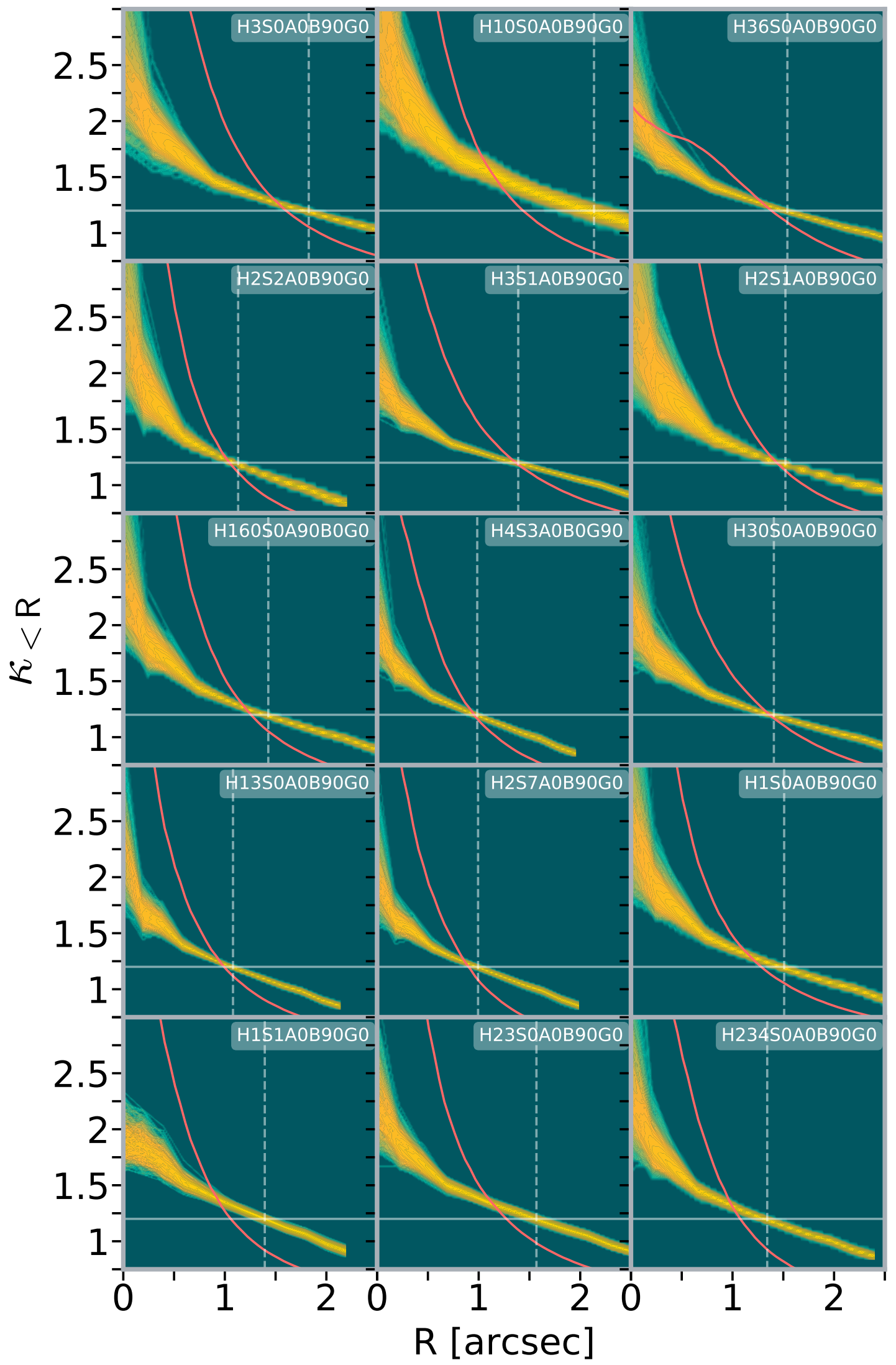

Figure 7. Circularly averaged enclosed surface density profiles of all reconstructed lenses. In other words, the plots show the enclosed mass as a mean $\kappa_{<R}$ within a given projected radius from the lensing galaxies' center of mass. The ensemble is represented with a coloured region with a gradient from green to yellow indicating its number density. The vertical line shows the approximate Einstein radius of the ensemble average. The red line shows the same profile for the actual convergence map. The Einstein radii of quads are in good agreement with the SEAGLE lenses. It is harder to find the correct Einstein radius for doubles. 


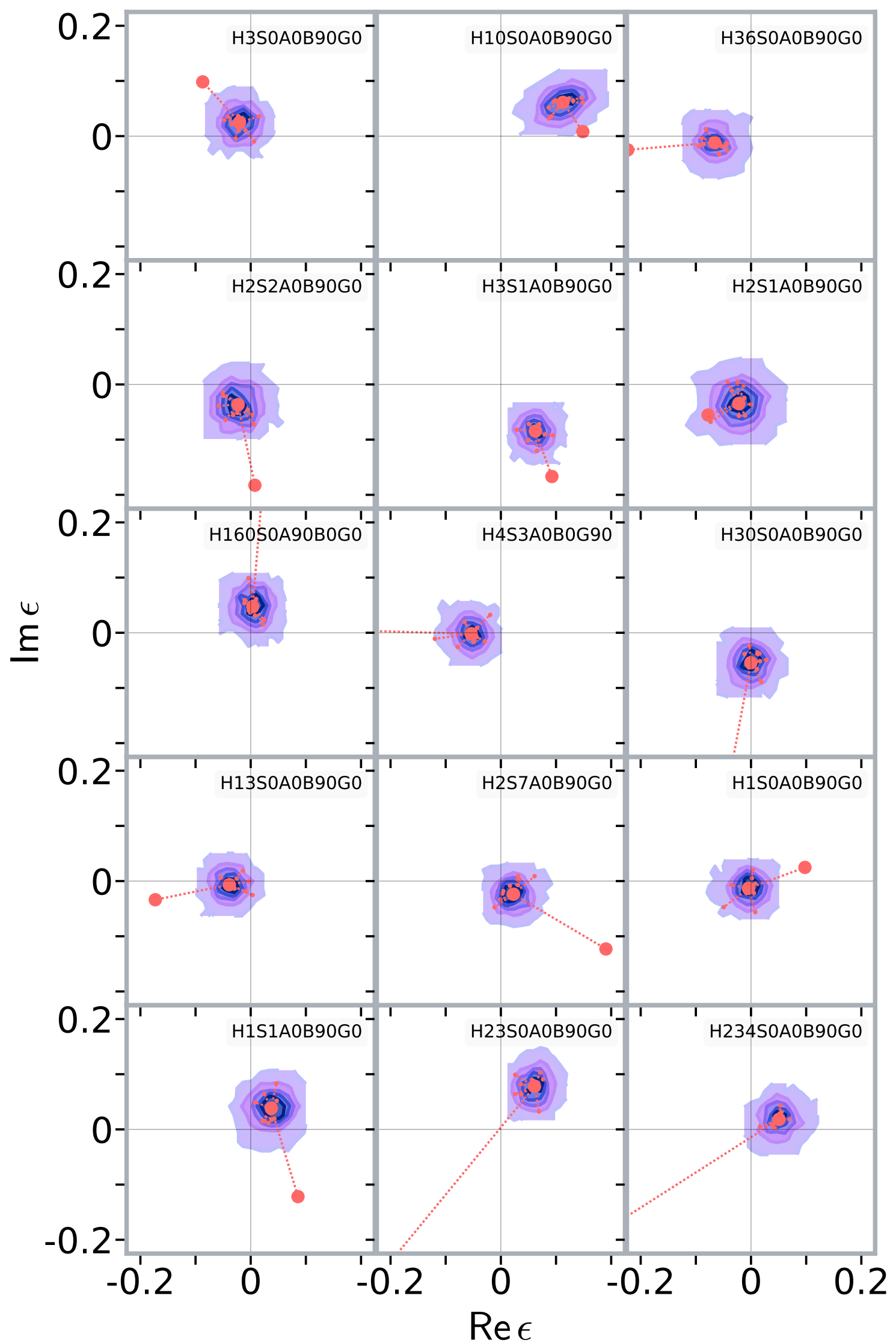

Figure 8. Complex ellipticity model distribution within each ensemble model, compared to the actual complex ellipticity of the SEAGLE-simulated lenses. The ellipticity of the SEAGLE lens is indicated with a large red dot which is connected to a graph with a center node indicated with another red dot representing the ellipticity of the model's ensemble average. The graphs leaf nodes are 20 randomly sampled points from the ensemble. In most cases, the models are too round, however their position angles roughly match. 

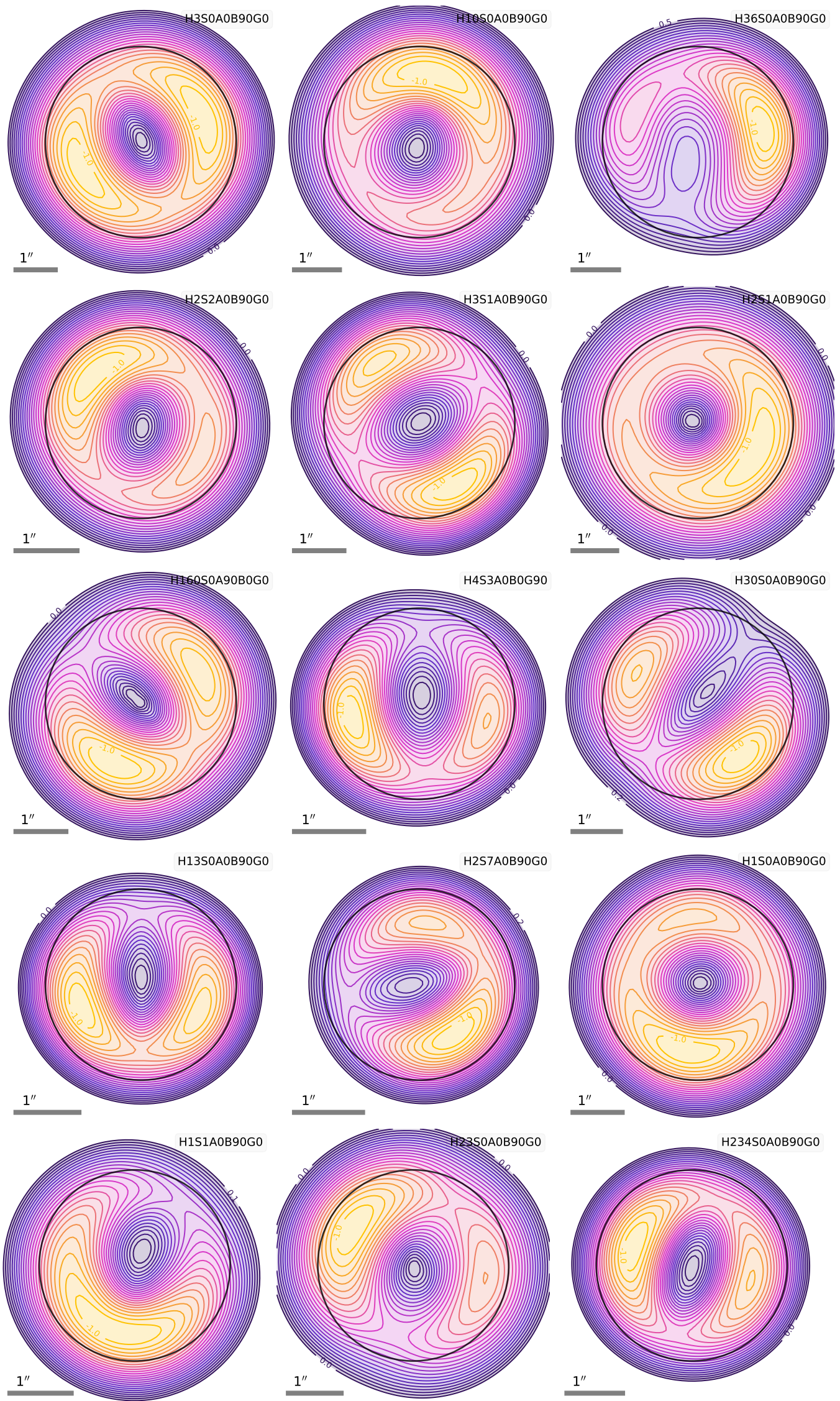

Figure 9. The actual Roche potentials of the SEAGLE-simulated lenses. The black circle indicates the area within which the scalar product (14) was evaluated. The potentials were shifted and scaled such that the center has a value of 0 , and the global minimum a value of -1 . This way, they offer a convenient way of comparing different models with the effect of the steepness degeneracy taken out. 


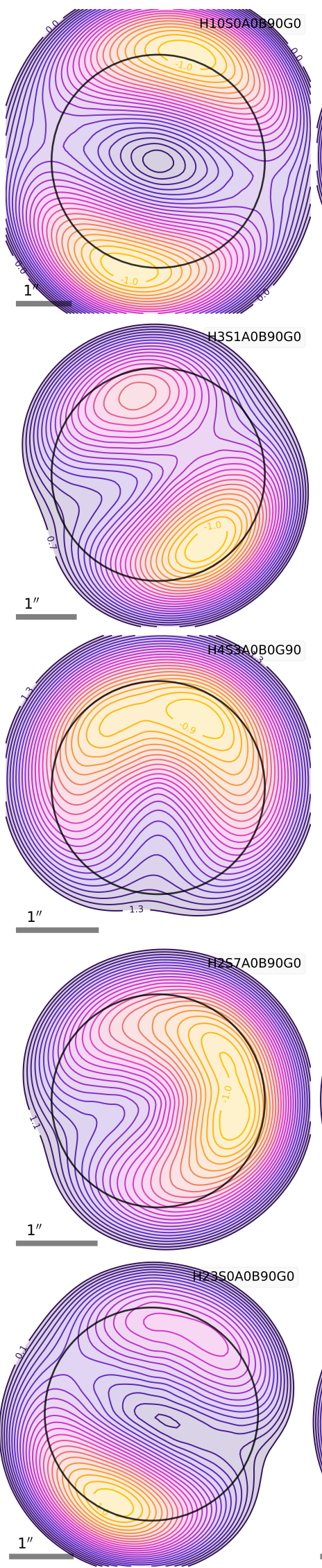
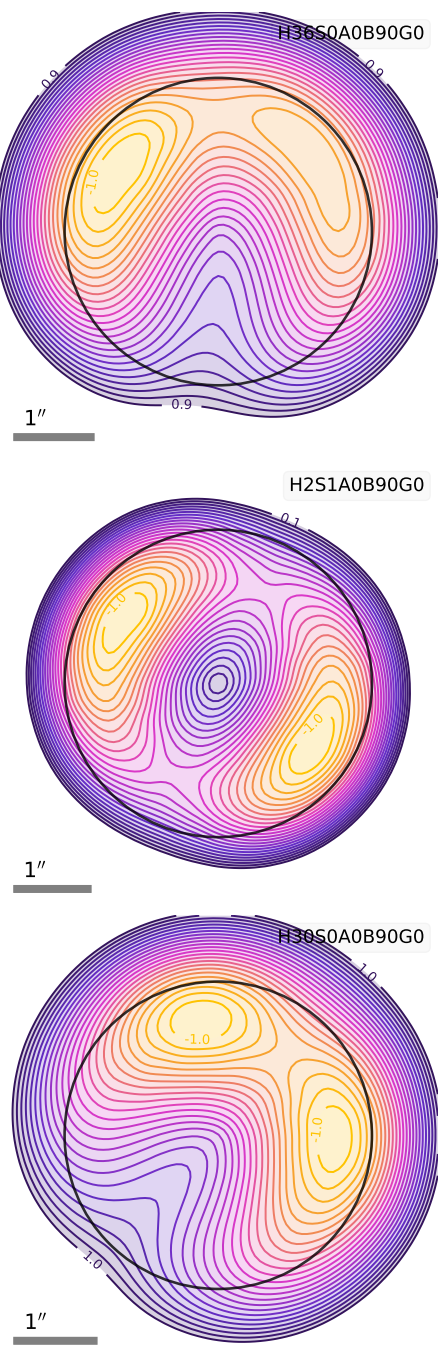

H1SOAOB90GO
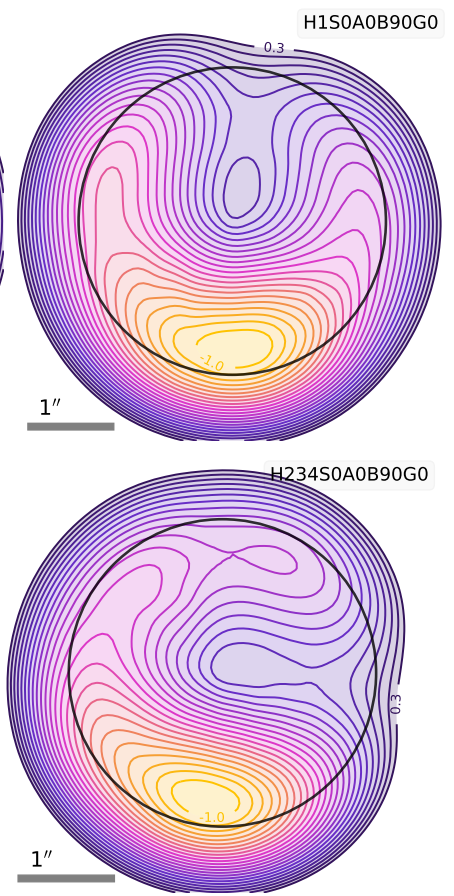

Figure 10. The Roche potentials of the 15 reconstructed lenses using the ensemble averaged model. All the scales are identical to the corresponding pictures in Figure 9. 


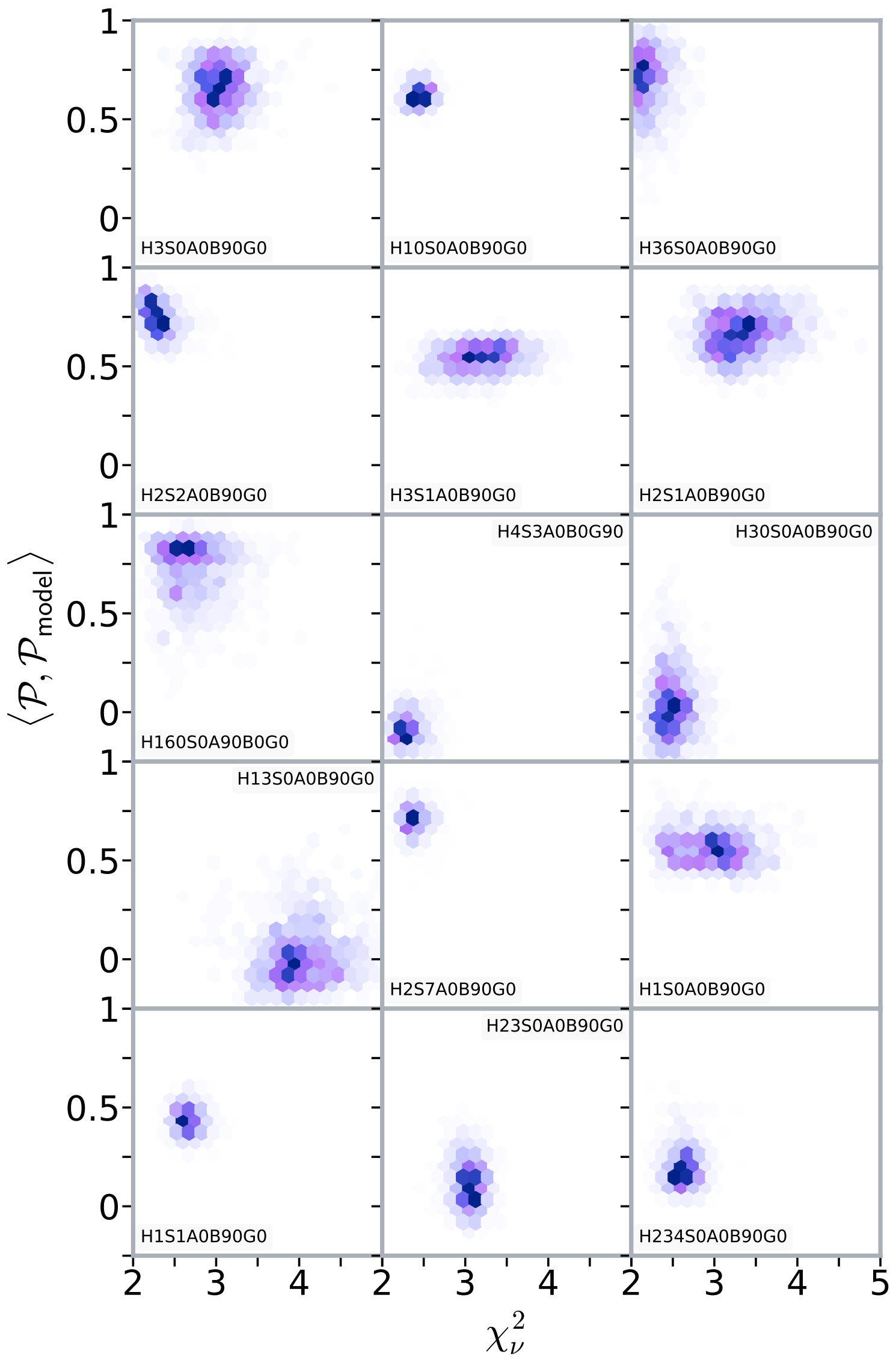

Figure 11. Hex-binned plots of distributions of reduced $\chi_{\nu}^{2}$ from the lens image reconstructions againts the distribution of the scalar product of Roche potentials for each ensemble model, see equations (11) and (14). Particularly good lens recovery is indicated by a scalar product close to 1 and a low $\chi^{2}$. 

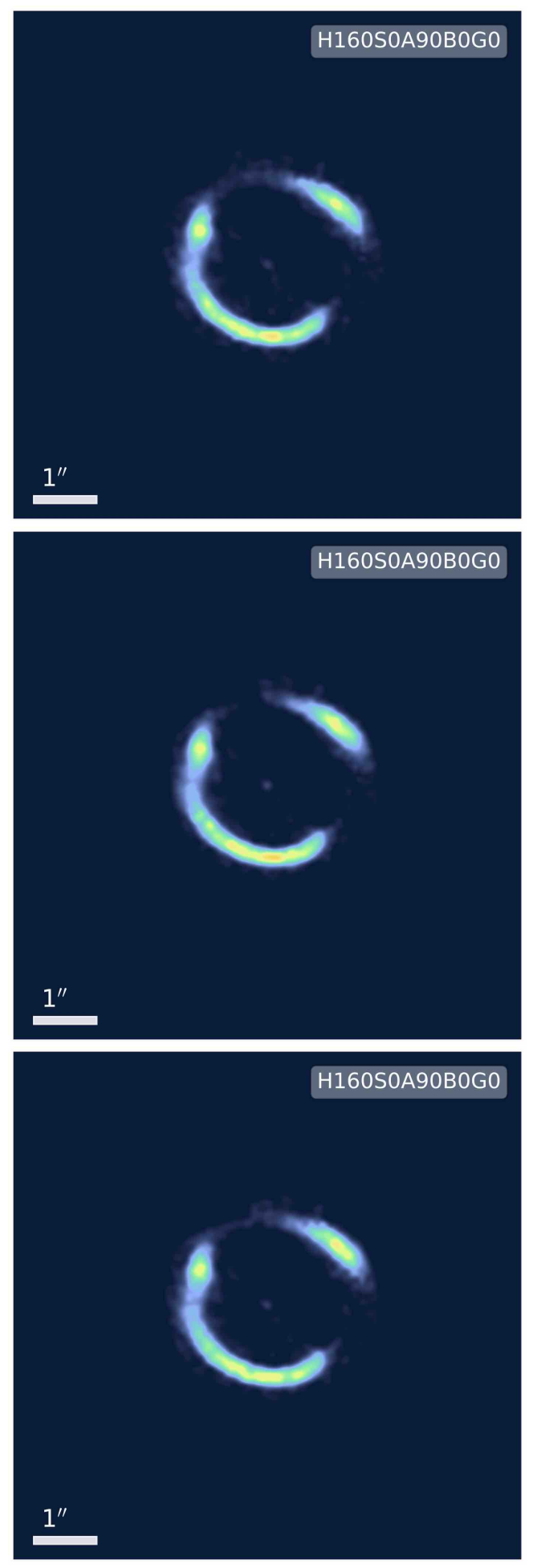

Figure 12. Alternative synthetic images of the lens H160S0A90B0G0 (cf. middle-left panel in Figure 3). Here, the image on top was generated from the unfiltered ensemble consisting of 1000 models. The middle shows the image generated with an ensemble containing 100 models with lowest $\chi^{2}$, and the bottom image shows the synthetic image from an ensemble containing 79 models which is the intersection of the set of lowest $\chi^{2}$ and the scalar products of the Roche potentials closest to 1 . The middle image shows a visible, albeit small, improvement compared to the top image, whereas the bottom image only shows slightly better relative brightness between the lens images compared to the middle, but no discernible improvement otherwise. 